\title{
Percutaneous Superficial Medial Collateral Ligament Release Outcomes During Medial Meniscal Arthroscopy: A Systematic Review
}

\author{
Michael A. Gaudiani, B.A., Derrick M. Knapik, M.D., Matthew W. Kaufman, B.A., \\ Michael J. Salata, M.D., James E. Voos, M.D., and Michael R. Karns, M.D.
}

\begin{abstract}
Purpose: To systematically review the literature to better understand the technique, outcomes, and complications after percutaneous superficial medial collateral ligament (sMCL) lengthening during knee arthroscopy to address isolated medial meniscal pathology. Methods: A systematic review was conducted according to the Preferred Reporting Items for Systematic Reviews and Meta-analyses (PRISMA) guidelines using a PRISMA checklist. The inclusion criteria consisted of English-language articles or articles with English-language translations documenting the use of percutaneous sMCL lengthening during arthroscopic knee surgery to treat isolated meniscal pathology (repair vs meniscectomy) with reported postoperative outcomes and complications. Results: Four studies met the inclusion criteria, consisting of a total of 192 patients undergoing percutaneous SMCL lengthening. No perioperative complications related to iatrogenic chondral damage, fracture, or additional meniscal injury were reported. Mild postoperative pain at the medial needle tract site lasting up to 15 days after surgery was reported in $52 \%$ of patients (46 of 88 ). At final follow-up, laxity on valgus stress testing showed a range from 0 to $1.1 \mathrm{~mm}$ with a range from $-0.3^{\circ}$ to $0.9^{\circ}$ of radiographic medial joint space widening compared with preoperative radiographs. The length of follow-up ranged from 1.5 to 24 months. Conclusions: The percutaneous "pie-crusting" technique remains the most commonly reported technique to lengthen the sMCL during arthroscopic meniscal surgery. Percutaneous lengthening represents a safe and effective method of increasing medial joint space visualization, with no reported perioperative or postoperative complications and with minimal, likely clinically insignificant residual joint laxity after surgery on valgus stress testing at final follow-up compared with preoperative values. Level of Evidence: Level IV, systematic review of Level IV studies.
\end{abstract}

$\mathbf{I}$ $\mathrm{n}$ the knee, the meniscus functions as a shock absorber, effectively distributing the load transmitted within the knee while ensuring joint stability by providing joint congruity between the femur and tibia throughout flexion and extension. Meniscal tearing

From the School of Medicine, Case Western Reserve University (M.A.G., M.W.K.); and University Hospitals Sports Medicine Institute (D.M.K., M.J.S., J.E.V., M.R.K.), Cleveland, Ohio, U.S.A.

The authors report the following potential conflicts of interest or sources of funding: M.J.S. receives personal fees from Stryker and Smith $\theta$ Nephew. J.E.V. is a paid consultant for Arthrex. M.R.K. receives personal fees from DePuy. Full ICMJE author disclosure forms are available for this article online, as supplementary material.

Received July 15, 2019; accepted October 21, 2019

Address correspondence to Michael A. Gaudiani, B.A., School of Medicine, Case Western Reserve University, 2109 Adelbert Rd, Cleveland, OH 44106, U.S.A.E-mail:mgaudiani@gmail.com

(C) 2019 THE AUTHORS. Published by Elsevier Inc. on behalf of the Arthroscopy Association of North America. This is an open access article under the CC BY-NC-ND license (http://creativecommons.org/licenses/by-nc-nd/4.0/). 2666-061X/19887

https://doi.org/10.1016/j.asmr.2019.10.009 represents one of the most commonly encountered pathologies in the knee, with a reported incidence as high as 6 per 1,000 persons in the United States alone. ${ }^{1}$ Owing to the loss of effective load transmission, untreated meniscal tears may lead to articular cartilage damage and eventual loss, resulting in the development of early osteoarthritic changes within the joint. ${ }^{2}$ As such, meniscal lesions are generally treated arthroscopically by primary repair or meniscectomy. In knees with tight medial compartments, particularly varus knees, the potential for missing meniscal pathology or misunderstanding the tear configuration because of poor visualization and iatrogenic chondral and meniscal injury is increased..$^{3-8}$ Although manipulation using a valgus force on the knee may help visualization, the surgeon must recognize the potential for medial collateral ligament (MCL) rupture or fracturing of the femur. ${ }^{5,9,10}$

The MCL is a viscoelastic structure that becomes stiff in the arthritic knee. ${ }^{11,12}$ To facilitate arthroscopic visualization of the medial compartment and meniscus, 
various MCL lengthening techniques have been described. Percutaneous, inside-out lengthening, or "pie crusting," first described by Agneskirchner and Lobenhoffer, ${ }^{13}$ represents a minimally invasive technique involving repeated puncturing of the medial capsuloligamentous structures with an intramuscular needle to lengthen the superficial medial collateral ligament (sMCL). ${ }^{14}$ Variants of the traditionally described pie-crusting method include an inside-out technique in which the needle is inserted through the anteromedial arthroscopy portal to lengthen the deep $\mathrm{MCL}^{15}$ and release of the sMCL using an open approach with subperiosteal stripping. ${ }^{16}$ With results from the percutaneous technique being the most commonly reported, concerns for iatrogenic MCL rupture, residual laxity, saphenous nerve injury, postoperative instability, and other potential complications after lengthening exist and warrant evaluation.

The purpose of this study was to systematically review the literature to better understand the technique, outcomes, and complications after percutaneous sMCL lengthening during knee arthroscopy to address isolated medial meniscal pathology. We hypothesized that patients would possess excellent outcomes with a low rate of postoperative valgus laxity and complications.

\section{Methods}

A systematic review was conducted according to the Preferred Reporting Items for Systematic Reviews and Meta-analyses (PRISMA) guidelines using a PRISMA checklist. ${ }^{11}$ All literature related to SMCL lengthening during arthroscopic knee surgery to treat medial meniscal pathology, published from January 1990 to May 2019, was identified. Two reviewers (M.G., D.K.) independently conducted the search in June 2019 using the following databases: PubMed, Biosis Previews, SPORTSDiscus, PEDro, and Embase. Each search included the following terms: meniscus AND medial AND arthroscopy AND meniscectomy AND repair AND medial collateral ligament AND release AND lengthening AND percutaneous AND open AND superficial.

The inclusion criteria consisted of English-language articles or articles with English-language translations documenting the use of percutaneous sMCL lengthening to increase medial joint space visualization during arthroscopic knee surgery to treat isolated medial meniscal pathology (repair vs meniscectomy) with reported postoperative outcomes and complications. The exclusion criteria were as follows: non-English-language studies; studies reporting lengthening techniques in total knee arthroplasty, deep MCL lengthening using an arthroscopic "insideout" approach, and open sMCL lengthening by periosteal stripping; studies performed in cadaveric specimens; biomechanical studies; patients undergoing medial meniscal surgery with concurrent treatment of concomitant injuries within the knee; studies not documenting the sMCL lengthening technique; or no recording of postoperative outcomes or complications after lengthening.

After the 2 independent authors' searches of the databases, a total of 47 citations were identified. The search process is shown in the flow diagram in Figure 1. After title and abstract assessment, a total of 16 full-text articles were selected for further evaluation. To ensure that all available studies were identified, references within each of the included articles were crossreferenced for inclusion if they were overlooked during the initial search. Statistical analysis was used to analyze reported postoperative clinical outcomes. All statistical analyses were performed using SPSS software (version 25.0; IBM, Armonk, NY).

\section{Results}

Of the 16 studies available for full-text review, 12 were excluded after initial literature review because of lack of reported postoperative outcomes $(n=6)$, use of cadaveric specimens $(n=2)$, techniques using deep MCL lengthening $(\mathrm{n}=2)$ or open sMCL lengthening via periosteal stripping $(\mathrm{n}=1)$, and lack of an Englishlanguage translation of the article $(n=1)$. A total of 4 studies were identified for analysis after application of the inclusion and exclusion criteria.

The 4 studies meeting the inclusion criteria consisted of a total of 192 patients undergoing percutaneous sMCL lengthening ${ }^{7,17-19}$ (Table 1). Men comprised 92\% of patients (118 of 128); patient sex was not recorded in a single study. ${ }^{7}$ Overall patient age at the time of surgery ranged from 19 to 62 years. Arthroscopic meniscectomy was performed in $76 \%$ of patients (146 of 192), whereas meniscal repair was performed in $24 \%$ (46 of 192). No perioperative complications related to iatrogenic chondral damage, fracture, or additional meniscal injury were reported. Postoperative bracing was used in 2 studies, ${ }^{7,19}$ for a mean of 4 weeks after lengthening, whereas 2 studies reported no postoperative bracing. ${ }^{17,18}$ Two studies reported the presence of mild postoperative pain at the medial needle tract site lasting up to 15 days after surgery in $40 \%$ of patients (28 of 70$).^{7,17}$ No incidences of saphenous nerve or saphenous vein injury were recorded.

The length of final follow-up ranged from 1.5 to 24 months after surgery. At final follow-up, laxity on valgus stress testing showed a range from 0 to 1.1 $\mathrm{mm}^{7,18,19}$ with a range from $-0.3^{\circ}$ to $0.9^{\circ 18,19}$ of radiographic medial joint space widening compared with preoperative radiographs. No cases of subjective instability were reported. The Lysholm score at final follow-up ranged from 85 to 94 , and no postoperative complications were reported in patients. 


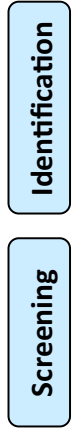

Figure 1. Preferred Reporting Items for Systematic Reviews and Meta-analyses (PRISMA) flowchart of study.

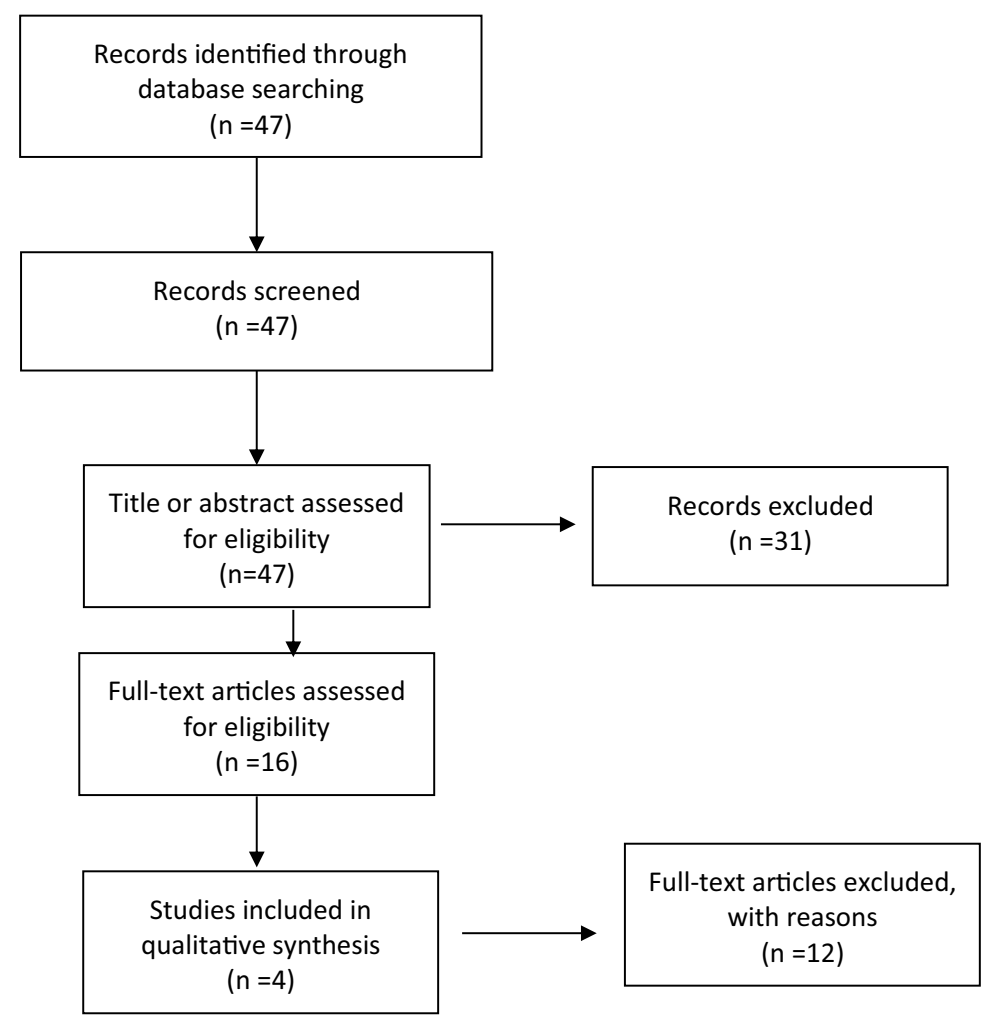

\section{Discussion}

The principal findings of this investigation were that in the current literature, the percutaneous pie-crusting technique was the most commonly performed technique, men were treated more often than women, patients' ages ranged from 19 to 62 years, and arthroscopic medial meniscectomy was more common than repair, with no iatrogenic damage to the chondral surface or meniscus reported. Bracing was used for a mean of 4 weeks after percutaneous lengthening in 2 studies, ${ }^{7,19}$ and pain at the medial tract site resolved in all patients by 15 days. At final follow-up, minimal residual joint laxity on valgus stress testing was reported compared with preoperative assessment, with no reports of subjective instability or postoperative complications.

The ages of patients undergoing knee arthroscopy with SMCL release ranged from 19 to 62 years, which encompasses a wide range and includes patients in whom mild to moderate osteoarthritic changes with joint space narrowing are expected. The development of degenerative changes within the knee coincides with stiffening of the knee, with prior studies showing a $184 \%$ increase in stiffness in the medial compartment of arthritic knees compared with nonarthritic knees. ${ }^{11,12}$ Degenerative changes along with anatomic variation can lead to a stiffer knee and smaller compartment. As a result, a tight medial joint space may lead to diagnostic errors with insufficient treatment or meniscal injury resulting in continued symptoms requiring revision surgery. ${ }^{20-24}$ Moreover, irreversible articular chondral injury, the most common complication after knee arthroscopy, is at higher risk of occurring in the setting of a tight medial compartment, accelerating degenerative changes in the knee..$^{25,26}$ Controlled lengthening of the sMCL allows for improve visualization and instrument access, with the percutaneous pie-crusting method being the most commonly used technique. ${ }^{7,15,17,27,28}$ Initially introduced by Agneskirchner and Lobenhoffer ${ }^{13}$ for softtissue balancing during total knee arthroscopy, the percutaneous technique relies on visualization using the anterior-lateral portal. ${ }^{19}$ Advantages of the percutaneous release technique include release without compromising the synovial membrane, decreasing the risk of intra-articular septic complications, ${ }^{17}$ while avoiding intra-articular structures that may impede instrument insertion required during the inside-out approach used to lengthen the deep MCL. ${ }^{14}$

Percutaneous, outside-in lengthening is preferable when addressing the sMCL as a result of the MCL possessing an extra-articular course around the knee. ${ }^{17}$ This technique involves perforating the SMCL near the posteromedial compartment with a hypodermic needle during arthroscopy. ${ }^{14}$ The biomechanical studies by Gardiner et al. ${ }^{10,29}$ showed that the posterior MCL, 
Table 1. Overview of Studies Included in Review

\begin{tabular}{|c|c|c|c|c|c|c|c|c|c|c|c|c|c|}
\hline Study & $\begin{array}{c}\text { Journal } \\
\text { (Year) }\end{array}$ & LOE & $\begin{array}{l}\text { No. of } \\
\text { Patients }\end{array}$ & $\begin{array}{c}\text { Age, } \\
\text { Mean } \pm \mathrm{SD} \\
\text { (Range), yr }\end{array}$ & Sex, n & Procedure, $\mathrm{n}$ & $\begin{array}{l}\text { Lengthening } \\
\text { Location }\end{array}$ & $\begin{array}{l}\text { Needle } \\
\text { Gauge }\end{array}$ & $\begin{array}{l}\text { Postoperative } \\
\text { Brace Use } \\
\text { (Duration, wk) }\end{array}$ & $\begin{array}{c}\text { Final } \\
\text { Follow-Up, } \\
\text { Mean } \\
\text { (Range), mo }\end{array}$ & $\begin{array}{c}\text { Valgus } \\
\text { Laxity, Mean* }\end{array}$ & $\begin{array}{c}\text { Outcomes, } \\
\text { Mean }+l-\text { SD }\end{array}$ & Complications \\
\hline $\begin{array}{c}\text { Lons } \\
\text { et al. }{ }^{18}\end{array}$ & $\begin{array}{l}\text { Orthop } \\
\quad \text { Traumatol } \\
\text { Surg Res } \\
(2018)\end{array}$ & IV & 40 & $39 \pm 9(20-54)$ & $\begin{array}{r}\text { M, 33 } \\
\text { F, } 7\end{array}$ & $\begin{array}{l}\text { Repair, } 7 \\
\text { Meniscectomy, } \\
33\end{array}$ & $\begin{array}{l}\text { Submeniscal, posterior } \\
\text { two-thirds }\end{array}$ & NR & No & 1.5 & $\begin{array}{l}+1.1 \pm 1.1 \mathrm{~mm} \\
\text { joint height } \\
+0.9^{\circ} \pm 1^{\circ} \\
\text { opening }\end{array}$ & $\begin{array}{l}\mathrm{NR} \\
\mathrm{n}\end{array}$ & None \\
\hline $\begin{array}{l}\text { Claret } \\
\quad \text { et al. } .^{17}\end{array}$ & Knee (2016) & IV & 70 & $44(19-62)$ & $\begin{array}{l}\text { M, } 44 \\
\quad \text { F, } 26\end{array}$ & $\begin{array}{l}\text { Meniscectomy, } \\
70\end{array}$ & $\begin{array}{l}\text { Synovial-meniscal } \\
\text { junction of posterior } \\
\text { horn }\end{array}$ & 20 & No & 6 & None & $\begin{array}{c}\text { Lysholm score, } \\
92 \pm 4 \\
\text { Tegner score, } 4 \\
\text { VAS score with } \\
\text { activity, 1 } \\
\text { VAS score at rest, 1 }\end{array}$ & None \\
\hline $\begin{array}{l}\text { Fakioglu } \\
\text { et al. }\end{array}$ & $\begin{array}{l}\text { Knee Surg } \\
\text { Sports } \\
\text { Traumatol } \\
\text { Arthrosc } \\
(2013)\end{array}$ & IV & 18 & $43(22-59)$ & NR & $\begin{array}{l}\text { Meniscectomy, } \\
18\end{array}$ & $\begin{array}{l}\text { Posterior third, proximal } \\
\text { to medial meniscus }\end{array}$ & 16 & Yes (4) & $8.3(6-12)$ & $\begin{array}{l}+0.1 \mathrm{~mm} \text { joint } \\
\text { height }\end{array}$ & Lysholm score, 94 & None \\
\hline $\begin{array}{l}\text { Jeon } \\
\text { et al. } .^{19}\end{array}$ & $\begin{array}{l}\text { Knee Surg } \\
\text { Sports } \\
\text { Traumatol } \\
\text { Arthrosc } \\
(2018)\end{array}$ & IV & 64 & $40.9 \pm 12.5$ & $\begin{array}{l}\text { M, } 41 \\
\quad \text { F, } 23\end{array}$ & $\begin{array}{l}\text { Repair, } 39 \\
\text { Meniscectomy, } \\
25\end{array}$ & $\begin{array}{l}\text { Posterior third, proximal } \\
\text { to medial meniscus }\end{array}$ & 19 & Yes (4) & 24 & $\begin{array}{c}0 \mathrm{~mm} \text { joint } \\
\text { height } \\
-0.3^{\circ} \\
\text { opening }\end{array}$ & $\begin{array}{c}\text { Lysholm score, } 85.1 \\
\pm 17.2 \\
\text { IKDC score, } 82.4 \pm \\
19.3 \\
\text { VAS score, } 1.1\end{array}$ & None \\
\hline
\end{tabular}

F, female; IKDC, International Knee Documentation Committee; LOE, level of evidence; M, male; NR, not recorded; SD, standard deviation; VAS, visual analog scale score.

${ }^{*}$ Measured on valgus stress testing at final follow-up and compared with preoperative stress radiograph values. 
proximal to the knee joint line, receives the highest strains with the knee in extension during an applied valgus load. In contrast, the cadaveric biomechanical study by Mihalko et al., ${ }^{30}$ comparing lengthening of the anterior half of the medial soft-tissue sleeve versus lengthening of the posterior half, reported improvement in medial joint space opening in knee flexion with anterior release. However, only 5 cadaveric specimens were used in each treatment group. All studies in our review reported lengthening along the posterior third of the MCL, warranting further study clinically evaluating the ideal location of SMCL lengthening to allow for adequate joint space opening while minimizing complications.

Release of the deep MCL has been less frequently reported. Park et al. ${ }^{27}$ reported using an inside-out technique to release the posterior aspect of the deep MCL to achieve adequate visualization of the posterior-horn footprint, allowing the proper working space required for posterior meniscal root repairs. This involves multiple needle punctures through the anteromedial portal under direct visualization of the posterior aspect of the ligament. ${ }^{15}$ Park et al. advocated deep MCL release because they concluded that preservation of the SMCL is essential to avoid medial instability postoperatively; however, no further justification for SMCL preservation was provided. Meanwhile, Li et al. ${ }^{6}$ reported that release of the deep MCL added minimal to no discomfort postoperatively without increased MCL excursion or valgus knee laxity postoperatively. In addition, Todor et al. ${ }^{14}$ indicated that the deep MCL stores most of the tension in the MCL and, as such, represents the proper structure to release tension. However, several biomechanical studies have identified the sMCL as the primary stabilizer against valgus forces, ${ }^{11,12,31}$ reported to supply $50 \%$ to $80 \%$ of the resistance to valgus stress on the knee. ${ }^{11}$ As such, although lengthening of the sMCL is more commonly reported, further studies comparing release of the SMCL and deep MCL regarding postoperative outcomes and complications are necessary to determine the optimal lengthening technique.

Lengthening of the SMCL has also been reported via subperiosteal stripping to release the distal attachment of the sMCL. Chung et al. ${ }^{16}$ evaluated 118 patients undergoing medial meniscal repair treated with postoperative bracing with a mean follow-up period of 42.4 \pm 19.3 months. They reported no intraoperative complications, whereas pain at the SMCL area was reported in $18 \%$ of patients $(21$ of 118 ) at 3 months and $7 \%$ of patients (8 of 118) at 6 months, with no patients reporting pain by 12 months or at final follow-up. Meanwhile, despite postoperative bracing for 6 weeks after surgery, $12 \%$ of patients possessed grade I laxity $\left(\leq 5^{\circ}\right.$ of valgus) at $30^{\circ}$ of flexion by 3 months postoperatively; $8 \%$, by 6 months; and $7 \%$, by 12 months. Although periosteal stripping represents a more invasive procedure, reflective of the increased duration of postoperative pain and laxity, further studies comparing the results of these techniques at mid- and long-term follow-up are needed.

No consensus currently exists on the necessity of postoperative bracing after arthroscopic meniscal repair or meniscectomy with concomitant SMCL release. Authors have reported that bracing in the setting of grade I or II MCL laxity is not necessary to prevent destabilization of the knee in the postoperative period. ${ }^{17,18}$ Meanwhile, Fakioglu et al. ${ }^{7}$ reported bracing postoperatively for ethical purposes given the lack of evidence regarding postoperative bracing, speculating that the use of the brace prevented objective instability in patients with grade I laxity. Meanwhile, Jeon et al. ${ }^{19}$ reported bracing as a prophylactic measure to prevent further injury to the MCL from valgus forces after lengthening. Although the MCL possesses an abundant vascular supply, allowing for good healing potential after injury, ${ }^{32,33}$ the impact of postoperative bracing on potential injury risk after SMCL lengthening remains unknown.

Despite no reported perioperative and postoperative complications, potential disadvantages of the percutaneous technique include injury to the saphenous nerve and vein., 17,28 However, such injuries are rarely reported when proper lengthening is performed, given that the cadaveric study by Roussignol et al. ${ }^{28}$ using 10 anatomic specimens found the saphenous nerve and vein to be remote from the percutaneous needle site in the medial knee. In addition, the reported degree of postoperative laxity was minimal, with no patient complaining of laxity requiring prolonged immobilization using a brace or evaluation for possible ligamentous rupture. Fakioglu et al. ${ }^{7}$ reported that compared with preoperative medial joint space width on valgus stress radiographs, mean laxity was significantly greater at 1 week $(+2 \mathrm{~mm})$ and 3 months $(+0.9 \mathrm{~mm})$ after surgery but decreased to $0.1 \mathrm{~mm}$ by 6 months Although no statistical comparison regarding postoperative joint laxity based on the use of postoperative bracing could be performed, further study is required, especially concerning the sustained laxity reported after periosteal stripping. ${ }^{16}$

\section{Limitations}

This study is not without limitations. Because of the strict inclusion and exclusion criteria, only 4 articles were identified and included. The small sample size and inherent heterogeneity of reported data limited our ability to conduct any meaningful statistical analyses assessing differences in outcomes between studies using percutaneous SMCL lengthening. Moreover, the limited number of investigations examining alternative SMCL techniques limited any analyses on outcomes between techniques. Outcomes and complications related to sMCL lengthening after arthroscopic knee surgery 
performed for the treatment of chondral, bony, or ligamentous injuries were not examined in this study, limiting the generalizability of our results only to patients undergoing arthroscopic treatment for medial meniscal pathology.

\section{Conclusions}

The percutaneous pie-crusting technique remains the most commonly reported technique to lengthen the sMCL during arthroscopic meniscal surgery. Percutaneous lengthening represents a safe and effective method of increasing medial joint space visualization, with no reported perioperative or postoperative complications and with minimal, likely clinically insignificant residual joint laxity after surgery on valgus stress testing at final follow-up compared with preoperative values.

\section{References}

1. Metcalf MH, Barrett GR. Prospective evaluation of 1485 meniscal tear patterns in patients with stable knees. Am J Sports Med 2004;32:675-680.

2. Nha KW, Lee YS, Hwang DH, et al. Second-look arthroscopic findings after open-wedge high tibia osteotomy focusing on the posterior root tears of the medial meniscus. Arthroscopy 2013;29:226-231.

3. Bosch U. Percutaneous perforation of the posteromedial capsuloligamentous structures to avoid cartilaginous damage due to arthroscopic intervention at the medial meniscal posterior horn in narrow joints. Oper Orthop Traumatol 2006;18:481-484 [in German].

4. Salzer M, Lin A, Miller CD, Herold S, Irrgang JJ, Harner CD. Complications after arthroscopic knee surgery. Am J Sports Med 2013;42:292-296.

5. Allum R. Complications of arthroscopy of the knee. J Bone Joint Surg Br 2002;84:937-945.

6. Li X, Selby RM, Newman A, O'Brien SJ. Needle assisted arthroscopic clysis of the medial collateral ligament of the knee: A simple technique to improve exposure in arthroscopic knee surgery. Orthop Rev (Pavia) 2013;5:38.

7. Fakioglu O, Ozsoy MH, Ozdemir HM, Yigit H, Cavusoglu AT, Lobenhoffer P. Percutaneous medial collateral ligament release in arthroscopic medial meniscectomy in tight knees. Knee Surg Sports Traumatol Arthrosc 2013:21:1540-1545.

8. Thorlund JB, Hare KB, Lohmander LS. Large increase in arthroscopic meniscus surgery in the middle-aged and older population in Denmark from 2000 to 2011. Acta Orthop 2014;85:287-292.

9. Huber W, Trieb K. Serious consequences of the wrong diagnosis of meniscal lesion in a case of stress fracture of the distal femur. Arthroscopy 2002;18:935-938.

10. Gardiner J, Weiss J, Rosenberg T. Strain in the human medial collateral ligament during valgus loading of the knee. Clin Orthop Relat Res 2001;391:266-274.

11. Grood ES, Noyes FR, Butler DL, Suntay WJ. Ligamentous and capsular restraints preventing straight medial and lateral laxity in intact human cadaver knees. J Bone Joint Surg Am 1981;63:1257-1269.

12. Griffith CJ, LaPrade RF, Johansen S, Armitage B, Wijdicks C, Engebretsen L. Medial knee injury: Part 1, static function of the individual components of the main medial knee structures. Am J Sports Med 2009;37: 1762-1770.

13. Agneskirchner J, Lobenhoffer P. Arthroscopic meniscus surgery technical operative methods. Unfallchirurg 2004;107:795-801 [in German].

14. Todor A, Caterev S, Nistor DV. Outside-in deep medial collateral ligament release during arthroscopic medial meniscus surgery. Arthrosc Tech 2016;5:e781-e785.

15. Atoun E, Debbi R, Lubovsky O, Weiler A, Debbi E, Rath E. Arthroscopic trans-portal deep medial collateral ligament pie-crusting release. Arthrosc Tech 2013;2:e41-e43.

16. Chung KS, Ha JK, Ra HJ, Kim JG. Does release of the superficial medial collateral ligament result in clinically harmful effects after the fixation of medial meniscus posterior root tears? Arthroscopy 2017;33:199-208.

17. Claret G, Montañana J, Rios J, et al. The effect of percutaneous release of the medial collateral ligament in arthroscopic medial meniscectomy on functional outcome. Knee 2016;23:251-255.

18. Lons A, Boureau F, Drumez E, Pasquier G, Putman S. Does medial collateral ligament pie-crusting induce residual laxity in arthroscopic management of medial meniscus tears? A prospective study of 40 cases. Orthop Traumatol Surg Res 2018;104:707-711.

19. Jeon SW, Jung M, Chun YM, et al. The percutaneous piecrusting medial release during arthroscopic procedures of the medial meniscus does neither affect valgus laxity nor clinical outcome. Knee Surg Sports Traumatol Arthrosc 2018;26:2912-2919.

20. Bin SI, Kim JM, Shin SJ. Radial tears of the posterior horn of the medial meniscus. Arthroscopy 2004;20:373-378.

21. Gillquist J, Hagberg G. A new modification of the technique of arthroscopy of the knee joint. Acta Chir Scand 1976;142:123-130.

22. Gillquist J, Hagberg G, Oretorp N. Arthroscopic examination of the posteromedial compartment of the knee joint. Int Orthop 1979;3:13-18.

23. Spahn G. Arthroscopic revisions in failed meniscal surgery. Int Orthop 2003;27:378-381.

24. Tolin B, Sapega A. Arthroscopic visual field mapping at the periphery of the medial meniscus: A comparison of different portal approaches. Arthroscopy 1993;9:265-271.

25. Buckwalter JA. Articular cartilage injuries. Clin Orthop Relat Res 2002;402:21-37.

26. Dick W, Glinz W, Henche HR, Ruckstuhl J, Wruhs O, Zollinger H. Complications of arthroscopy. A review of 3714 cases (author's transl). Arch Orthop Trauma Surg 1978;92:69-73 [in German].

27. Park YS, Moon HK, Koh YG, et al. Arthroscopic pullout repair of posterior root tear of the medial meniscus: The anterior approach using medial collateral ligament piecrusting release. Knee Surg Sports Traumatol Arthrosc 201 1;19:1334-1336.

28. Roussignol X, Gauthe R, Rahali S, Mandereau C, Courage O, Duparc F. Opening the medial tibiofemoral 
compartment by pie-crusting the superficial medial collateral ligament at its tibial insertion: A cadaver study. Orthop Traumatol Surg Res 2015;101:529-533.

29. Gardiner J, Weiss J. Subject-specific finite element analysis of the human medial collateral ligament during valgus knee loading. J Orthop Res 2001;21:1098-1106.

30. Mihalko WM, Woodard EL, Hebert CT, Crockarell JR, Williams JL. Biomechanical validation of medial piecrusting for soft-tissue balancing in knee arthroplasty. J Arthroplasty 2015;30:296-299.
31. Kennedy J, Fowler P. Medial and anterior instability of the knee. An anatomical and clinical study using stress machines. Clin Orthop Relat Res 1995;321:3-9.

32. Miyamoto R, Bosco JA, Sherman OH. Treatment of medial collateral ligament injuries. J Am Acad Orthop Surg 2009;17:152-161.

33. Wijdicks BCA, Griffith CJ, Johansen S, Engebretsen L. Injuries to the medial collateral ligament and associated medial structures of the knee. J Bone Joint Surg Am 2010;92:1266-1280. 